\title{
Unified modelling of granular media with Smoothed Particle Hydrodynamics
}

\author{
Chong Peng ${ }^{1} \cdot$ Xiaogang Guo ${ }^{1,2} \cdot$ Wei $\mathrm{Wu}^{1}\left(\mathbb{D} \cdot\right.$ Yongqi Wang $^{2}$
}

Received: 17 March 2016/Accepted: 9 September 2016/Published online: 20 September 2016

(C) The Author(s) 2016. This article is published with open access at Springerlink.com

\begin{abstract}
In this paper, we present a unified numerical framework for granular modelling. A constitutive model capable of describing both quasi-static and dynamic behaviours of granular material is developed. Two types of particle interactions controlling the mechanical responses, frictional contact and collision, are considered by a hypoplastic model and a Bagnold-type rheology relation, respectively. The model makes no use of concepts like yield stress or flow initiation criterion. A smooth transition between the solid-like and fluid-like behaviour is achieved. The Smoothed Particle Hydrodynamics method is employed as the unified numerical tool for both solid and fluid regimes. The numerical model is validated by simulating element tests under both quasi-static and flowing conditions. We further proceed to study three boundary value problems, i.e. collapse of a granular pile on a flat plane, and granular flows on an inclined plane and in a rotating drum.
\end{abstract}

Keywords Bagnold rheology · Granular material · Hypoplasticity $\cdot$ Rotating drum $\cdot$ Smoothed Particle Hydrodynamics · Solid/fluid transition

Wei Wu

wei.wu@boku.ac.at

1 Institut für Geotechnik, Universität für Bodenkultur, Feistmantelstrasse 4, 1180 Vienna, Austria

2 Fachgebiet für Strömungsdynamik FB Maschinenbau, Technische Universität Darmstadt, Otto-Berndt-Strasse 2, 64287 Darmstadt, Germany

\section{Introduction}

Granular materials are widely involved in various industry processes and natural phenomena. In industry, one is often interested in the granular flow capacity through complex geometry without blockage [32], as well as the forces exerting on structures [13]. In the field of geophysics, landslides and debris flows are natural hazards possessing great threat to human society. It is therefore important to predict the flowing mass, velocity, run-out distance and impact force of these hazards on structures. An appropriate constitutive model and an adequate numerical method capable of providing high-quality simulation of granular media are of great interest.

As granular materials are collections of rigid macroscopic particles, the discrete element method (DEM), based on elementary mechanical principals idealized from particle interactions [14, 47], is considered suitable for granular modelling. However, the DEM traces the motion and mechanical response of each particle, which makes the computational cost still prohibitive in real-scale industrial and geophysical simulations. On the other hand, in traditional continuum approaches, granular materials are considered as continuum media and can be described using constitutive models and field variables. Therefore, the reliability of continuum approaches largely depends on how exact the constitutive models are in portraying the granular behaviours.

It is well known that, depending on the geometry and loading condition, a granular body can behave like a solid or liquid [22, 15]. Many works have been carried out to study the granular behaviours in the two distinct regimes. Theories of soil mechanics, e.g. elastoplasticity and hypoplasticity, capture the salient behaviours of granular 
materials in the quasi-static solid-like regime. Constitutive models for the quasi-static regime are usually rate-independent, making them inappropriate for the modelling of granular flows. Rate-dependent viscoplastic models consider viscous strain in the post-failure regime and usually describe a progressive evolution from plastic flow to viscous flow [39]. However, previous studies show that a granular flow can happen without satisfying the failure condition $[39,16,25]$ and sometimes is characterized by an abrupt solid/fluid transition [18, 2]. On the other hand, models using a viscous relation based on fluid dynamics are widely used to simulate the flow regime. For instance, non-Newtonian rheology finds its application in granular flow modelling [20, 40]. Another example of granular rheology, more elaborated and sophisticated, is the $\mu(I)$ model based on dimension analysis and experimental observations [2, 24]. The $\mu(I)$ model draws much attention recently in granular flow simulations [11, 46, 5]. Usually, in numerical simulations, a granular rheology is used together with a yield stress. Around the yield stress, the viscosity changes drastically from a low physical value to an artificial numerical one. The issue for granular rheology is that the quasi-static solid material behaviours inside the yield surface are undefined.

In industrial and geophysical problems, the granular flows often consist of flowing and stagnant regions. In these two regions, the material can be described with the models mentioned above from soil mechanics and fluid dynamics, respectively. A straightforward approach is to model the two regimes with different material models. This method requires complex numerical techniques making use of different solvers for solid and fluid. Identification of the solid/fluid interfaces is also necessary. Moreover, the solid/ fluid transition needs to be explicitly given to capture the evolution of solid/fluid interfaces. Therefore, a more rational way is to unify the solid-like and fluid-like responses as well as the solid/fluid transition in one single constitutive model. A few efforts have been proposed $[1,17,9]$, by including rate-dependency in conventional elastoplastic models. In these models, flows can happen only after yielding. One noteworthy model is presented in $[39,38]$, which is a combination of an elastoplastic model and a Bingham rheology. In this model, viscous flows can be activated before the plastic limit criterion being reached by checking the second-work instability [16]. Nevertheless, the development of well-established unified relations describing both solid-like and fluid-like behaviours of granular materials is still an ongoing undertaking.

Granular flow modelling also has some special requirements for numerical methods. An eligible method should be capable of simulating problems of solid mechanics and fluid dynamics at the same time with an acceptable complexity. Both large deformation of solids and free surface flow of fluids need to be modelled. Conventional grid-based numerical methods such as finite element method (FEM) and finite differential method (FDM) have difficulty in taking such task. Recently developed particle-based methods, e.g. Smoothed Particle Hydrodynamics (SPH), material point method (MPM) and particle FEM (PFEM), are more suitable for granular modelling. Nevertheless, previous studies apply either rate-independent models for quasi-static state $[7,36,30]$, or pure rheology models for flow regime [11]. Unified numerical modelling of granular media still calls for further investigation.

In this paper, we present a unified modelling of granular media using the SPH method. A rational yet simple constitutive relation capable of describing solid-like, fluid-like and solid/fluid transition behaviours of granular materials is adopted. The constitutive model makes use of a hypoplastic model and an extended 3-D Bagnold-type relation. It captures the failure and critical state properties of granular materials in the quasi-static regime, as well as the viscous behaviour and collision-induced dilatation in dynamic granular flows. The meshfree SPH method is employed as it proves applicable in both large deformation analysis and fluid dynamics. SPH kernel gradient renormalization is applied to treat the particle inconsistency, thus improve the accuracy. We first validate the unified method with element tests. Some granular flow problems with common configuration are then numerically simulated.

\section{A unified constitutive model for granular materials}

\subsection{Constitutive model framework}

Two main interactions between individual granular particles are friction and collision [43, 8]. In the quasi-static regime, the interparticle frictional forces give rise to rateindependent Coulomb-type plastic behaviour. On the other hand, in the dynamic flow state, a notable part of stress comes from the moment transfer in the interparticle collision. This collision-induced stress is rate-dependent. A unified model should consider the friction and collision behaviours within a single framework. Previous works on unified models usually make use of viscoplastic models $[1,38]$, where a decomposition in the granular kinematics is assumed, i.e. the strain is decomposed into elastic, plastic and viscous parts. In this work, we employ a decomposition of stress rather than strain, namely

$\boldsymbol{\sigma}=\boldsymbol{\sigma}_{\text {friction }}+\boldsymbol{\sigma}_{\text {collision }}$

where $\boldsymbol{\sigma}$ is the Cauchy stress tensor, the subscripts 'friction' and 'collision' indicate corresponding stress parts, respectively. The two stress parts take their effects simultaneously, 
regardless of the flow state. Namely, the model makes no explicit distinction between quasi-static state and dynamic flow state. The solid/fluid transition is realized by the coupled evolution of the two stress parts. To obtain a concrete formulation, several restrictions should be imposed on the constitutive equation (1). Firstly, the rate-dependent part $\sigma_{\text {collision }}$ should vanish automatically or be negligibly small in the quasi-static state, as there is little particle collision. Secondly, the frictional part $\boldsymbol{\sigma}_{\text {friction }}$ should cease to exist in granular gasification, where the collision is the dominate interaction. Thirdly, the resulting constitutive relation should be able to capture the salient behaviours of granular materials both in quasi-static and flow regimes.

A hypoplastic model and an extended Bagnold-type rheology are employed to formulate the unified model. For this purpose, Eq. (1) is rewritten as

$\sigma=\sigma_{\mathrm{h}}+\sigma_{\mathrm{v}}$

where $\sigma_{\mathrm{h}}$ and $\sigma_{\mathrm{v}}$ denote the hypoplastic (frictional contact) and dynamic viscous (collision) stress parts, respectively.

\subsection{Quasi-static solid-like response}

Hypoplastic constitutive models are based on nonlinear tensorial functions with the major advantage of simple formulation and few parameters. In this paper. we will embark on the first hypoplastic model with critical state proposed by $\mathrm{Wu}$ et al. [51]. Recently, some improved hypoplastic models have been available [27, 45], which aim to improve the dependence of stiffness on pressure and density. However, such models are rather complex in formulation with more model parameters. Since the initiation of granular flow is mainly dictated by failure rather than deformation, the original model by $\mathrm{Wu}$ et al. with its simple formulation and few parameters offers a better choice for the unified modelling of granular media.

Let $\dot{\boldsymbol{\varepsilon}}=\left[\nabla \boldsymbol{v}+(\nabla \boldsymbol{v})^{\mathrm{T}}\right] / 2$ be the strain rate tensor, the hypoplastic model with critical state [51] has the following form

$$
\begin{aligned}
\stackrel{\circ}{\boldsymbol{h}}^{=} & c_{1}\left(\operatorname{tr} \boldsymbol{\sigma}_{\mathrm{h}}\right) \dot{\boldsymbol{\varepsilon}}+c_{2} \frac{\operatorname{tr}\left(\boldsymbol{\sigma}_{\mathrm{h}} \dot{\boldsymbol{\varepsilon}}\right) \boldsymbol{\sigma}_{\mathrm{h}}}{\operatorname{tr} \boldsymbol{\sigma}_{\mathrm{h}}} \\
& +\left(c_{3} \frac{\boldsymbol{\sigma}_{\mathrm{h}} \boldsymbol{\sigma}_{\mathrm{h}}}{\operatorname{tr} \boldsymbol{\sigma}_{\mathrm{h}}}+c_{4} \frac{\boldsymbol{\sigma}_{\mathrm{h}}^{*} \boldsymbol{\sigma}_{\mathrm{h}}^{*}}{\operatorname{tr} \boldsymbol{\sigma}_{\mathrm{h}}}\right)\|\dot{\boldsymbol{\varepsilon}}\| I_{e}
\end{aligned}
$$

In the above equation, $\operatorname{tr}(\cdot)$ denotes the trace of the enclosed tensor, $\|\dot{\boldsymbol{\varepsilon}}\|=\sqrt{\operatorname{tr}(\dot{\boldsymbol{\varepsilon}}: \dot{\boldsymbol{\varepsilon}})}$ stands for the Euclidean norm, and $\boldsymbol{\sigma}_{\mathrm{h}}^{*}=\boldsymbol{\sigma}_{\mathrm{h}}-\left(\operatorname{tr} \boldsymbol{\sigma}_{\mathrm{h}} / 3\right) \boldsymbol{I}$ is the deviatoric part of the hypoplastic stress tensor. The four material parameters $c_{1}$, $c_{2}, c_{3}$ and $c_{4}$ can be obtained from laboratory tests or calculated from the commonly used material parameters: initial elastic moduli $E_{i}$, initial Poisson ratio $\mu_{i}$, frictional angle $\phi$ and dilation angle $\psi$ [50]. $\stackrel{\circ}{\mathrm{h}}_{\mathrm{h}}$ is the Jaumann stress rate used to keep frame objectivity in large deformation, which is defined as

$\stackrel{\circ}{\boldsymbol{\sigma}}_{\mathrm{h}}=\dot{\boldsymbol{\sigma}}_{\mathrm{h}}+\boldsymbol{\sigma}_{\mathrm{h}} \dot{\boldsymbol{\omega}}-\dot{\boldsymbol{\omega}} \sigma_{\mathrm{h}}$

where $\dot{\boldsymbol{\sigma}}_{\mathrm{h}}$ is the hypoplastic Cauchy stress rate, and the spin tensor $\dot{\boldsymbol{\omega}}$ is defined as $\dot{\boldsymbol{\omega}}=\left[\nabla \boldsymbol{v}-(\nabla \boldsymbol{v})^{\mathrm{T}}\right] / 2$.

The hypoplastic model (3) takes the critical state concept into consideration by using the density function $I_{e}$

$I_{e}=(a-1) D_{c}+1$

where $a$ is a material constant and $D_{c}$ is the modified relative density

$$
D_{c}=\frac{e_{\mathrm{crt}}-e}{e_{\mathrm{crt}}-e_{\mathrm{min}}}
$$

where $e, e_{\min }$ and $e_{\mathrm{crt}}$ are the initial, minimum and critical void ratio, respectively. Based on laboratory tests, the critical void ratio $e_{\mathrm{crt}}$ and material constant $a$ can be related to the stress level [51]

$$
a=q_{1}+q_{2} \exp \left(q_{3} \operatorname{tr} \boldsymbol{\sigma}_{\mathrm{h}}\right), \quad e_{\mathrm{crt}}=p_{1}+p_{2} \exp \left(p_{3} \operatorname{tr} \boldsymbol{\sigma}_{\mathrm{h}}\right)
$$

The parameters $p_{1} \sim p_{3}$ and $q_{1} \sim q_{3}$ can be obtained by fitting the experimental data.

The constitutive model (3) captures the salient solid-like behaviours of granular material, such as nonlinearity in stress-strain relation, loading path dependence, stress level dependence of friction angle and dilatancy and critical state behaviour [51]. It covers the whole spectrum of granular from loose to dense packing. Unlike elastoplastic models, the employed model makes no distinction between the elastic and plastic ranges. The strain decomposition and complex stress integration algorithms are unnecessary when using the hypoplastic model. Therefore, numerical implementation can be greatly simplified. It is noteworthy that although the hypoplastic model is developed without recourse to plasticity theory, some widely-accepted concepts, such as failure surface and flow rule, may be obtained as natural outcomes [52-54]. Figure 1 shows the failure surface and flow rule derived from the hypoplastic model. The failure surface in 3-D principal stress space is a conical surface, close to the failure surface of MohrCoulomb plastic criterion but without singularities. The plastic flow directions in Fig. 1b indicate that the flow rule of the employed hypoplastic model is non-associative.

\subsection{Extended 3-D Bagnold-type rheology}

Many rheology models have been used in granular flow modelling, from simple Bingham model $[39,38,26]$ to more sophisticated ones [24, 46, 1]. These models consider the combined frictional and collisional interactions from a phenomenological point of view by a single viscosity 


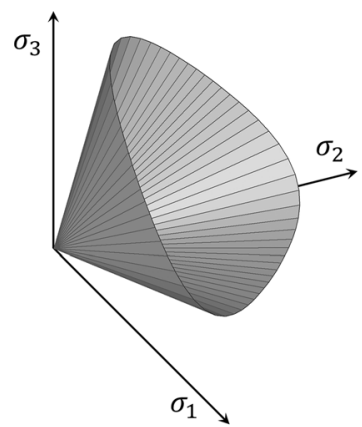

(a)

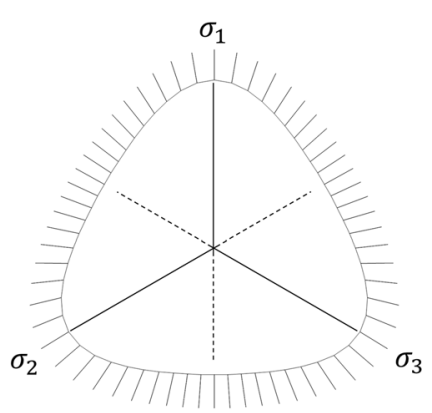

(b)
Fig. 1 Failure surface and flow rule derived from the constitutive Eq. (3): a failure surface; $\mathbf{b}$ flow rule on $\pi$-plane

coefficient. In our model, the dynamic part only accounts for the collision interaction; therefore, the viscous relations mentioned above are inappropriate.

Bagnold [4] studied a gravity-free dispersion of wax spheres sheared in Newtonian fluids and found that the shear stress-strain relation falls into two ranges, i.e. the macro-viscous range and grain-inertia range. In the macroviscous range, the viscosity of interstitial fluids affects the granular shear stress significantly. However, it is observed that the shear stress in grain-inertia range is independent of the interstitial fluid viscosity. According to [4, 44, 3], the grain-inertia range corresponds to fast shear stage in the experiments, where the particle collision dominates the bulk behaviour and dissipation of the flow kinetic energy. Experiments [4, 3] and theoretical analyses [41, 23] suggest a quadratic dependence of shear stress on shear strain rate in a simple shear configuration

$$
\tau_{x z}=k_{\mathrm{v}}\left(\frac{\mathrm{d} v_{x}}{\mathrm{~d} z}\right)^{2}
$$

where $k_{\mathrm{v}}$ is a coefficient related to particle size, density and volume fraction, and $x, z$ are Cartesian coordinates. Moreover, a collision-induced pressure $p_{\mathrm{v}}$, termed dispersive pressure, is found proportional to the shear stress in rapid shearing $[4,3]$

$p_{\mathrm{v}}=\tau_{x z} / \tan \alpha_{i}$

where $\tan \alpha_{i}$ denotes the ratio between the shear stress and dispersive pressure in the grain-inertia fluid range, and $\alpha_{i}$ is a material constant. As the above relations are obtained in very high shear rates without confining pressure, they can be viewed as mechanical responses caused solely by particle collisions. Therefore, these relations are adopted in the unified model to account for the particle collision in the flowing state.

The simple shear models (8) and (9) need to be extended to tensorial form for 3-D problems. A general form for nonNewtonian fluids may take the form $[43,48]$ $\boldsymbol{\sigma}_{v}=a_{1} \boldsymbol{I}+a_{2} \dot{\boldsymbol{\varepsilon}}$

where $\sigma_{v}$ is the dynamic stress part in Eq. 2 and $\boldsymbol{I}$ stands for the unit tensor. The coefficients are

$a_{i}=f_{i}\left(I_{D}, I I_{D}, I I I_{D}, C, \nabla C\right), \quad i=1,2$

where $I_{D}=\operatorname{tr}(\dot{\boldsymbol{\varepsilon}}), I I_{D}=\left[(\operatorname{tr} \dot{\boldsymbol{\varepsilon}})^{2}-\operatorname{tr}\left(\dot{\boldsymbol{\varepsilon}}^{2}\right)\right] / 2$ and $I I I_{D}=\operatorname{det} \dot{\boldsymbol{\varepsilon}}$ are three invariants of the strain rate tensor and $C$ is the solid volume fraction. Following the general form, we propose an extended Bagnold-type rheology for granular flows

$\boldsymbol{\sigma}_{v}=\left(-\frac{4 k_{\mathrm{v}}}{\tan \alpha_{i}}\left\|\dot{\boldsymbol{\varepsilon}}^{*}\right\|\right) \boldsymbol{I}+\left(4 k_{\mathrm{v}} \sqrt{\left\|\dot{\boldsymbol{\varepsilon}}^{*}\right\|}\right) \dot{\boldsymbol{\varepsilon}}^{*}$

where $\dot{\boldsymbol{\varepsilon}}^{*}=\dot{\boldsymbol{\varepsilon}}-(\operatorname{tr} \dot{\boldsymbol{\varepsilon}} / 3) \boldsymbol{I}$ is the deviatoric part of the strain rate tensor. It can easily be shown that for a simple shear flow this constitutive model is reducible to the relations Eqs. (8) and (9). According to Eq. (12), the dynamic stress tensor $\boldsymbol{\sigma}_{v}$ consists of an isotropic pressure part and a pure shear stress part. The origin of these two stress parts is that there are two types of kinetic energy in rapid granular flows, respectively, the kinetic energy associated with the main velocity and the fluctuation of individual particle velocity. The mean kinetic energy is in the direction of the granular flow, resulting to the shear stress tensor in Eq. (12). On the other hand, the kinetic energy associated with random velocity fluctuations is isotropic, which gives rise to the dispersive pressure. In fast flows dominating by particle collision, these two stress parts can be related by a constant $\alpha_{i}[4,3]$.

\subsection{The unified model for granular materials}

Combining the frictional and collisional contributions Eqs. (3) and (12), we obtain the following unified constitutive model

$\boldsymbol{\sigma}=\int \dot{\boldsymbol{\sigma}}_{\mathrm{h}} \mathrm{d} t+\left(-\frac{4 k_{\mathrm{v}}}{\tan \alpha_{i}}\left\|\dot{\boldsymbol{\varepsilon}}^{*}\right\|\right) \boldsymbol{I}+\left(4 k_{\mathrm{v}} \sqrt{\left\|\dot{\boldsymbol{\varepsilon}}^{*}\right\|}\right) \dot{\boldsymbol{\varepsilon}}^{*}$

The integral on the right-hand side of Eq. (13) is the history-dependent hypoplastic stress part for the frictional contacts, while the remaining parts are the rate-dependent stress part for the collisions in rapid granular flows. We couple these two stress parts directly, because the dynamic rate-dependent part is negligibly small in the quasi-static state, and the frictional part ceases in rapid flows dominated by particle collision. We will show in Sect. 4.2 that in certain circumstances, i.e. sand liquefaction and granular gasification, the contribution of the hypoplastic stress part virtually vanishes.

In general granular problems where particle interactions possibly include friction and collision, both the stress parts 
take effect simultaneously. The proportions of frictional and collisional stresses depend on the kinematics, loading and boundary conditions of flows. For instance, from very slow motion to rapid flow, the dynamic part becomes more and more significant. This results in an increase in the dispersive pressure $p_{v}$ and a tendency of dilation, thus leads to a relaxation in the normal stress of frictional contact. Therefore, the hypoplastic stress part tends to decrease with the increase of shear rate. This dynamic balance can be achieved in numerical simulations.

The presented model bridges the granular solid-like and fluid-like behaviours and achieves a natural solid/fluid transition without any flow initiation criterion. The frictional and collisional stress parts evolve concurrently depending on the flow state, the loading and boundary conditions. Complex concepts such as yield stress and strain decomposition are not required in the model. Therefore, the numerical implementation is simplified. The unified model has 13 material parameters: $c_{1} \sim c_{4}$ related to hypoplasticity, $e_{\min }, q_{1} \sim q_{3}$ and $p_{1} \sim p_{3}$ for critical state, and two parameters $\alpha_{i}$ and $k_{\mathrm{v}}$ for the dynamic part. According to [4], the parameter $k_{\mathrm{v}}$ can be further calculated from particle density, particle diameter and solid volume fraction.

\section{SPH modelling of granular material}

Granular flows often involve large deformation from solid mechanics point of view and free surface flow from a fluid dynamics perspective, which are cumbersome to handle with conventional numerical methods. The meshfree and Lagrangian properties of SPH make it an appealing method for granular flow modelling [11, 7, 36, 37, 12].

\subsection{The governing equations}

In this paper, only dry granular flows are considered. Therefore, the interstitial fluid is ignored and the granular material is modelled as single phase medium. The governing equations for granular flows in the Lagrangian description take the form

$$
\begin{aligned}
& \frac{\mathrm{d} \rho}{\mathrm{d} t}=-\rho \nabla \cdot \boldsymbol{v} \\
& \frac{\mathrm{d} \boldsymbol{v}}{\mathrm{d} t}=\frac{1}{\rho} \nabla \boldsymbol{\sigma}+\boldsymbol{g}
\end{aligned}
$$

where $\mathrm{d}(\cdot) / \mathrm{d} t$ is the material derivative, $\rho$ is the density of the continuous medium, and $g$ is the body force. The governing equations consist of conservation of mass (14) and momentum (15).

\subsection{The SPH formulations}

The SPH is a Lagrangian meshfree method with no computational grid. The whole computational domain $\Omega$ is discretized with a set of particles, which carry the physical properties and computational variables, and move with material velocities. By tracking the movement of the particles and the evolution of the carried variables, the considered problem can be solved numerically. Because the SPH applies an updated Lagrangian formulation, large deformation problems in solid mechanics as well as free surface tracking in fluid dynamics are treated naturally. It is therefore an excellent choice for the numerical modelling of granular media, where the material may come across large deformation in quasi-static motion, and rapid free surface flow after failure.

We refer $\boldsymbol{x}=(x, y, z)$ as a spatial point. In the SPH method, a field function $f(\boldsymbol{x})$ is approximated by the following convolution integral

$$
\langle f(\boldsymbol{x})\rangle=\int_{\Omega} f\left(\boldsymbol{x}^{\prime}\right) W\left(\boldsymbol{x}-\boldsymbol{x}^{\prime}, h\right) \mathrm{d} \boldsymbol{x}^{\prime}
$$

The kernel function $W\left(\boldsymbol{x}-\boldsymbol{x}^{\prime}, h\right)$ is a compact-supported weighting function, where $h$ is a smoothing length used to control the support size of $W$. The kernel function used through this paper is a Wendland $C^{6}$ function $[19,49]$ for which the compact support has a radius of $2 h$. The field function's spatial derivatives are obtained by substituting $\nabla f(\boldsymbol{x})$ into Eq. (16)

$$
\langle\nabla f(\boldsymbol{x})\rangle=-\int_{\Omega} f\left(\boldsymbol{x}^{\prime}\right) \nabla W \mathrm{~d} \boldsymbol{x}^{\prime}
$$

In SPH discretization, each particle $i$ has an associated kernel function $W_{i}$ centred at $\boldsymbol{x}_{i}$. Replacing the continuous integrations in Eqs. (16) and (17) with particle summation, the discrete approximations of the field function and its derivatives are written as

$$
\begin{aligned}
& f\left(\boldsymbol{x}_{i}\right)=\sum_{j=1}^{n} f\left(\boldsymbol{x}_{j}\right) W_{i j} \frac{m_{j}}{\rho_{j}} \\
& \nabla f\left(\boldsymbol{x}_{i}\right)=\sum_{j=1}^{n} f\left(\boldsymbol{x}_{j}\right) \nabla_{i} W_{i j} \frac{m_{j}}{\rho_{j}}
\end{aligned}
$$

where $n$ is the number of particles within the support domain of particle $i$ and $m_{j} / \rho_{j}$ denotes the volume of material represented by particle $j$. Through SPH simulations, the mass of a particle is usually constant but its density can evolve according to the varying inter-particle spacing, resulting to constantly changing particle volume. $\nabla_{i} W_{i j}$ is the gradient of kernel function $W_{i j}$ evaluated at the location $\boldsymbol{x}_{i}$. 
Many particle discretization forms of the governing equations are available in the SPH literature. By numerical experiments, we find that the following expressions give rise to better results

$\frac{\mathrm{d} \rho_{i}}{\mathrm{~d} t}=\sum_{j=1}^{n} m_{j}\left(\boldsymbol{v}_{i}-\boldsymbol{v}_{j}\right) \cdot \nabla_{i} W_{i j}$

$\frac{\mathrm{d} \boldsymbol{v}_{i}}{\mathrm{~d} t}=\sum_{j=1}^{n}\left(\frac{\boldsymbol{\sigma}_{i}}{\rho_{i}^{2}}+\frac{\boldsymbol{\sigma}_{j}}{\rho_{j}^{2}}\right) \nabla_{i} W_{i j} m_{j}+\boldsymbol{g}_{i}$

where $\sigma$ is the total stress tensor, consisting of the hypoplastic stress part and the dynamic stress part.

The calculation of stress rate in the proposed constitutive relation requires the velocity gradient, which can be computed in the SPH as

$$
\nabla \boldsymbol{v}_{i}=\sum_{j=1}^{n}\left(\boldsymbol{v}_{j}-\boldsymbol{v}_{i}\right) \otimes \nabla_{i} W_{i j} \frac{m_{j}}{\rho_{j}}
$$

\subsection{The correction of kernel gradient}

The continuous SPH kernel interpolation in Eq. (16) theoretically ensures second-order accuracy for interior regions. That is, constant and linear functions can be reproduced exactly. However, this $C^{0}$ and $C^{1}$ consistency are not always satisfied in the SPH particle approximation, especially when particle distributions are irregular, or the particle support domain is truncated by boundaries [28]. This deficiency, termed particle inconsistency, is the direct cause of the relatively low accuracy and slow convergence rate in the original SPH method. Usually the lack of $C^{1}$ consistency is more hazardous, because the discrete forms of the governing Eqs. (20) and (21) all make use of the kernel gradient $\nabla W$. Many corrections have been proposed to improve the accuracy of kernel gradient approximation. In the present study, we use the renormalization technique $[6,34]$ to enforce the $C^{1}$ consistency, which is briefly given as follows.

The gradient of the field function $f(\boldsymbol{x})$ can be rewritten as

$$
\nabla f(\boldsymbol{x})=\nabla f(\boldsymbol{x})-f(\boldsymbol{x}) \nabla 1
$$

The continuous kernel approximation of the above equation reads

$$
\langle\nabla f(\boldsymbol{x})\rangle=\int_{\Omega} f\left(\boldsymbol{x}^{\prime}\right) \nabla W \mathrm{~d} \boldsymbol{x}^{\prime}-f(\boldsymbol{x}) \int_{\Omega} \nabla W \mathrm{~d} \boldsymbol{x}^{\prime}
$$

Performing the second-order Taylor expansion to the first term on the right-hand side of Eq. (24), we have

$$
\begin{aligned}
\int_{\Omega} f\left(\boldsymbol{x}^{\prime}\right) \nabla W \mathrm{~d} \boldsymbol{x}^{\prime}= & f(\boldsymbol{x}) \int_{\Omega} \nabla W \mathrm{~d} \boldsymbol{x}^{\prime} \\
& +\left(\int_{\Omega}\left(\boldsymbol{x}^{\prime}-\boldsymbol{x}\right) \otimes \nabla W \mathrm{~d} \boldsymbol{x}^{\prime}\right) \nabla f(\boldsymbol{x}) \\
& +O\left(h^{2}\right)
\end{aligned}
$$

Substituting the above equation into Eq. (24) gives

$$
\langle\nabla f(\boldsymbol{x})\rangle=\left(\int_{\Omega}\left(\boldsymbol{x}^{\prime}-\boldsymbol{x}\right) \otimes \nabla W \mathrm{~d} \boldsymbol{x}^{\prime}\right) \nabla f(\boldsymbol{x})+O\left(h^{2}\right)
$$

From Eq. (26), we can see that the kernel approximations of function gradient have second-order accuracy when the following requirement is satisfied:

$\int_{\Omega}\left(\boldsymbol{x}^{\prime}-\boldsymbol{x}\right) \otimes \nabla W \mathrm{~d} \boldsymbol{x}^{\prime}=\left(\begin{array}{ll}1 & 0 \\ 0 & 1\end{array}\right)$

In order to ensure $C^{1}$ consistency in the SPH method, the above requirement needs to be satisfied in discrete particle approximation. This is achieved using the renormalization technique, which employs the corrected kernel gradient $\nabla^{C} W$ such that at particle $i$

$\sum_{j=1}^{n}\left(\boldsymbol{x}_{j}-\boldsymbol{x}_{i}\right) \otimes \nabla_{i}^{C} W_{i j} \frac{m_{j}}{\rho_{j}}=\left(\begin{array}{ll}1 & 0 \\ 0 & 1\end{array}\right)$

where $\nabla_{i}^{C} W_{i j}=\boldsymbol{L}\left(\boldsymbol{x}_{i}\right) \nabla_{i} W_{i j}$ denotes the corrected kernel gradient at particle $i$. $\boldsymbol{L}$ is the renormalization matrix in the following form

$$
\boldsymbol{L}\left(\boldsymbol{x}_{i}\right)=\left(\begin{array}{ll}
\sum_{j=1}^{n}\left(x_{j}-x_{i}\right) \frac{\partial W_{i j}}{\partial x_{i}} \frac{m_{j}}{\rho_{j}} & \sum_{j=1}^{n}\left(x_{j}-x_{i}\right) \frac{\partial W_{i j}}{\partial y_{i}} \frac{m_{j}}{\rho_{j}} \\
\sum_{j=1}^{n}\left(y_{j}-y_{i}\right) \frac{\partial W_{i j}}{\partial x_{i}} \frac{m_{j}}{\rho_{j}} & \sum_{j=1}^{n}\left(y_{j}-y_{i}\right) \frac{\partial W_{i j}}{\partial y_{i}} \frac{m_{j}}{\rho_{j}}
\end{array}\right)^{-1}
$$

The corrected kernel gradient $\nabla W^{C}$ is applied to Eqs. (20), (21) and (22), replacing the original kernel gradient $\nabla W$. The employment of the corrected gradient kernel ensures $C^{1}$ consistency in the SPH method.

\subsection{Numerical implementation and boundary conditions}

In the SPH method, the solution of granular flow problems is obtained by marching Eqs. (20) and (21) forward in time starting from initial conditions. Many explicit integration methods are available for this task. In this paper, we employ a Predictor-Corrector scheme [21]. The time step of the explicit solver is controlled by a combination of the Courant condition and the maximum acceleration [36]

$\Delta t=\chi_{\mathrm{CFL}} \min \left(\frac{h}{\max \left\|\boldsymbol{a}_{i}\right\|}, \frac{h}{c}\right)$

where $a_{i}=\mathrm{d} v_{i} / \mathrm{d} t$ is the particle acceleration, $c$ is the artificial speed of sound used to control the size of the time step, and $\chi_{\mathrm{CFL}}$ is the Courant condition coefficient. In this paper, $c$ and $\chi_{\mathrm{CFL}}$ are taken as $80 \mathrm{~m} / \mathrm{s}$ and 0.05 , respectively. 
In the unified model, the hypoplastic stress part is history-dependent. Therefore, the hypoplastic stress tensor $\sigma_{\mathrm{h}}$ is calculated by integrating hypoplastic stress rate $\dot{\boldsymbol{\sigma}}_{\mathrm{h}}$ using the same Predictor-Corrector scheme. As long as the time step is small enough, the direct stress integration of the hypoplastic stress is accurate sufficiently [36]. Since there is no explicit failure in hypoplasticity, complex stress integration and return-mapping algorithms in elastoplasticity are unnecessary.

Two kinds of boundary conditions are considered in this paper, i.e. periodic boundary and non-slip solid boundary. The periodic boundary condition in SPH is straightforward, as shown in $[11,21]$. The treatment of solid boundary condition is still a challenge in SPH computations. In this paper, we employ a boundary particle method developed for geomechanical applications [35]. In this method, the solid boundary is discretized with boundary particles, which take part in the SPH approximation like real particles but keep fixed or move with prescribed motions. The velocity and stress tensor at boundary particles are extrapolated from the real granular particles. We find that this boundary treatment method works properly in our simulations.

\section{SPH element tests}

In this section, the proposed unified constitutive model and the SPH implementation are validated using two element tests. For simplicity, velocity restrictions are imposed on SPH particles to reproduce a uniform deformation condition in the test sample, analogous to an element test in the FEM.

\subsection{Quasi-static undrained simple shear test}

First we aim to test the unified model as well as the SPH method at the quasi-static range. An undrained simple shear test is simulated. It is widely known that saturated sand specimens with different void ratio demonstrate three types of stress-strain behaviour in undrained simple shear tests, i.e. liquefaction, limited liquefaction and dilation [10]. In real applications, the accurate modelling of these phenomena is crucial because it determines whether a subsequent material flow happens. The computational setup is shown in Fig. 2, with a size of $0.1 \times 0.1 \mathrm{~m}$. Periodic boundary conditions are applied to reproduce a uniform shear deformation, where arbitrary large shear strain can be modelled. The undrained condition is simulated by keeping the volume fixed. Pore water is not considered, so all stresses presented in the analysis are effective stress. The constitutive parameters given in Table 1 are obtained by referring to the experiments in [55]. The parameters $k_{\mathrm{v}}$

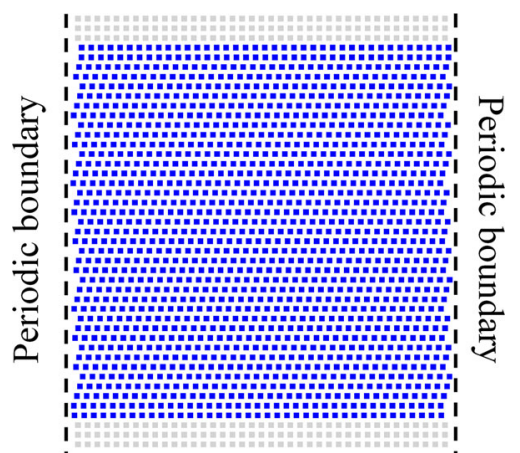

Fig. 2 Computational setup for the undrained simple shear test. Granular particles and boundary particles are marked blue and grey

and $\alpha_{i}$ for the flow range are assumed, because the experiments are only performed with quasi-static loading. Strictly speaking, $k_{\mathrm{v}}$ depends on the initial void ratio. However, the dynamic part is insignificant when shear rate is low, so a fixed $k_{\mathrm{v}}$ is used.

A velocity field of $v_{x}=0.01 z$ and $v_{z}=0 \mathrm{~m} / \mathrm{s}$ is imposed on the particles as boundary condition for the element test, which corresponds to a constant shear rate of $\dot{\varepsilon}_{x z}=0.5 \% /$ $\mathrm{s}$, larger than that in the experiments. However, with the applied shear rate the dynamic stress part is sufficiently small $\left(\sim 10^{-7} \mathrm{~Pa}\right)$, so numerically the simulation lies in the quasi-static range. Five simulations with different initial void ratios are performed with a confining pressure $p_{0}=100 \mathrm{kPa}$. The numerical results are given in Fig. 3 . For the loose specimens, i.e. $e=0.890$ and $e=0.865$, the strain-softening liquefaction can be observed. A residual stress smaller than the peak stress is obtained in large shear strain. At intermediate void ratio $e=0.845$, the stress in large shear is only slightly smaller than the peak stress, indicating a limited liquefaction. The specimens with lower initial void ratio show no peak behaviour and tend to dilate during shearing, resulting in a higher ultimate stress in large shear strain. From Fig. 3b, it can be seen all the specimens reach the failure line following different paths. The modelling successfully captures the sand behaviours in the quasi-static simple shear tests. Although the dynamic part is calculated as well, in the low shear rate condition it reduces to negligibly small value, thus the quasi-static problem can be properly modelled.

\subsection{Granular sheared in an annular shear cell}

In this section, the experiments by Savage and Sayed [44] with a cohesionless dry granular material sheared in an annular shear cell are modelled. The experiments aim to investigate the stress developed in rapid shearing dry granular material. In the experiments, the height of the specimens is maintained constant, corresponding to a fixed 
Table 1 Constitutive parameters used in the simple shear test

\begin{tabular}{|c|c|c|c|c|c|c|c|c|c|c|c|c|}
\hline $\begin{array}{l}c_{1} \\
(-)\end{array}$ & $\begin{array}{l}c_{2} \\
(-)\end{array}$ & $\begin{array}{l}c_{3} \\
(-)\end{array}$ & $\begin{array}{l}c_{4} \\
(-)\end{array}$ & $\begin{array}{l}e_{\min } \\
(-)\end{array}$ & $\begin{array}{l}p_{1} \\
(-)\end{array}$ & $\begin{array}{l}p_{2} \\
(-)\end{array}$ & $\begin{array}{l}p_{3} \\
\left(\mathrm{kPa}^{-1}\right)\end{array}$ & $\begin{array}{l}q_{1} \\
(-)\end{array}$ & $\begin{array}{l}q_{2} \\
(-)\end{array}$ & $\begin{array}{l}q_{3} \\
\left(\mathrm{kPa}^{-1}\right)\end{array}$ & $\begin{array}{l}k_{\mathrm{v}} \\
(-)\end{array}$ & $\begin{array}{l}\alpha_{i} \\
\left({ }^{\circ}\right)\end{array}$ \\
\hline-50.0 & -629.6 & -629.6 & 1220.8 & 0.597 & 0.53 & 0.45 & -0.0018 & 1.0 & -0.4 & -0.0001 & 0.01 & 35.0 \\
\hline
\end{tabular}

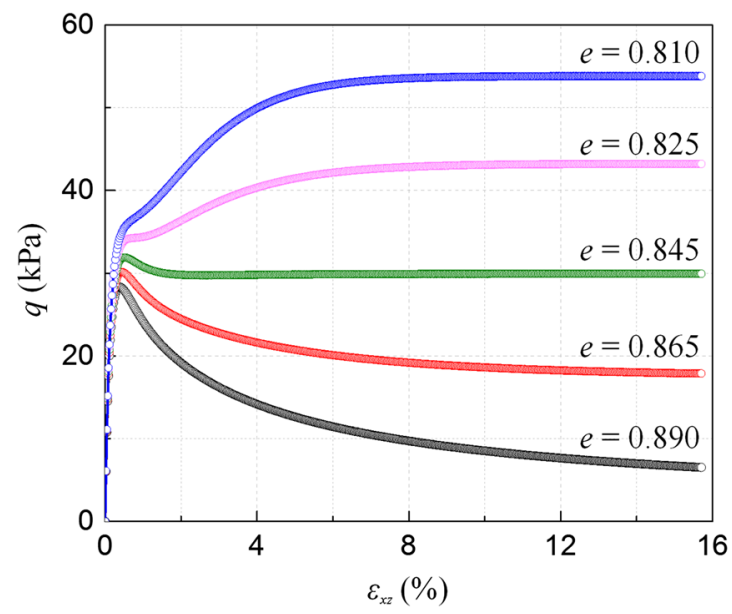

(a)

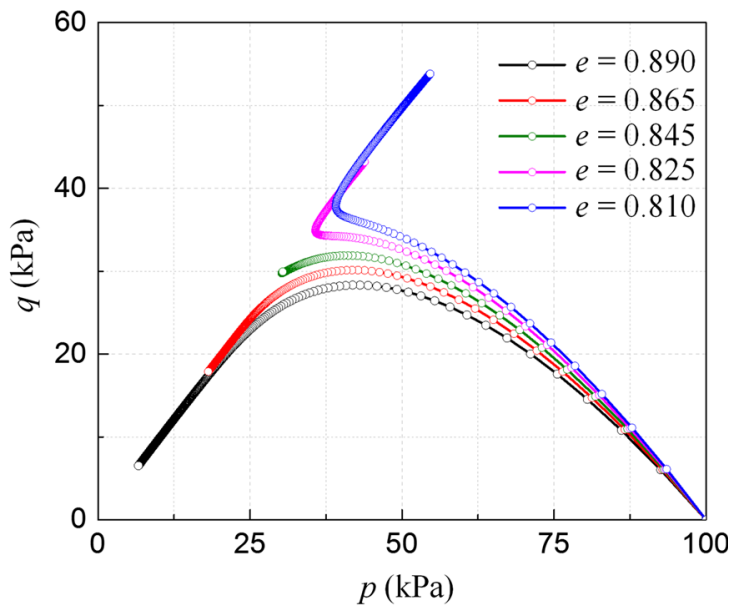

(b)

Fig. 3 Simple shear tests with different initial void ratios

Table 2 Constitutive parameters used in the annular shear test

\begin{tabular}{lllllllllll}
\hline $\begin{array}{l}c_{1} \\
(-)\end{array}$ & $\begin{array}{l}c_{2} \\
(-)\end{array}$ & $\begin{array}{l}c_{3} \\
(-)\end{array}$ & $\begin{array}{l}c_{4} \\
(-)\end{array}$ & $\begin{array}{l}e_{\min } \\
(-)\end{array}$ & $\begin{array}{l}p_{1} \\
(-)\end{array}$ & $\begin{array}{l}p_{2} \\
(-)\end{array}$ & $\begin{array}{l}p_{3} \\
\left(\mathrm{kPa}^{-1}\right)\end{array}$ & $\begin{array}{l}q_{1} \\
(-)\end{array}$ & $\begin{array}{l}q_{2} \\
(-)\end{array}$ & $\begin{array}{l}q_{3} \\
\left(\mathrm{kPa}^{-1}\right)\end{array}$ \\
\hline-50.0 & -746.6 & -746.6 & 1855.1 & 0.563 & 0.65 & 0.55 & -0.11 & 1.0 & -0.24 & -0.013 \\
\hline
\end{tabular}

volume condition in the numerical modelling. The initial normal stress ranges from 100 to $1500 \mathrm{~Pa}$ due to gravity, thus $p_{0}=500 \mathrm{~Pa}$ is chosen as the initial confining pressure in the numerical simulations. We consider the first group of experiments in [44], No. PS18 PS21 with small polystyrene beads. The physical properties of the beads are: particle density $\rho_{s}=1095 \mathrm{~kg} / \mathrm{m}^{3}$, diameter $d_{s}=1.0 \mathrm{~mm}$. According to the geometry of the experimental apparatus, the experiments can be modelled as an infinite simple shear test with fixed volume. Therefore, the computational setup displayed in Fig. 2 is again employed. The material parameters used in the numerical modelling is given in Table 2. Following the experiments, four simulations with different solid volume fraction (hence different void ratio) are performed. The solid volume fraction and corresponding void ratio are given in Table 3.

In the numerical simulations a velocity field of $v_{x}=2 \kappa z$ and $v_{z}=0 \mathrm{~m} / \mathrm{s}$ is imposed, where $\kappa$ is the desired shear rate. In this work, the range of shear rate $\kappa$ is $10 \sim 500$. At the beginning, the model is given a shear rate of $\kappa=10$ for $0.5 \mathrm{~s}$, to make sure that the granular flow fully develops and the critical state is reached. Then $\kappa$ is linearly increased to
500 in $20 \mathrm{~s}$. Due to the extremely large shear strain, any stress part from the hypoplastic model is the residual stress after critical state being reached.

Table 3 lists the residual stress from simulations with different solid volume fraction after the first $0.5 \mathrm{~s}$ of shearing. It can be seen that for $C=0.461, C=0.483$ and $C=0.504$, the residual stresses are zero, corresponding to a full liquefaction (or gasification since the interstitial fluid is air). It means that the granular particles lose frictional contact from a constitutive modelling point of view. For the dense case $C=0.524$, a nonzero residual stress is obtained regardless of the magnitude of shear strain. According to [4], viscous coefficient $k_{\mathrm{v}}$ can be calculated

Table 3 List of solid volume fraction $C$, void ratio $e$, residual stresses and viscous coefficient $k_{\mathrm{v}}$ in the four simulations

\begin{tabular}{lllll}
\hline Solid volume fraction $C$ & 0.461 & 0.483 & 0.504 & 0.524 \\
Void ratio $e$ & 1.169 & 1.070 & 0.984 & 0.908 \\
$\sigma_{x z}(\mathrm{~Pa})$ & 0.0 & 0.0 & 0.0 & 36.5 \\
$\sigma_{z z}(\mathrm{~Pa})$ & 0.0 & 0.0 & 0.0 & 83.2 \\
$k_{\mathrm{v}}$ & 0.0018 & 0.0027 & 0.0041 & 0.0065 \\
\hline
\end{tabular}




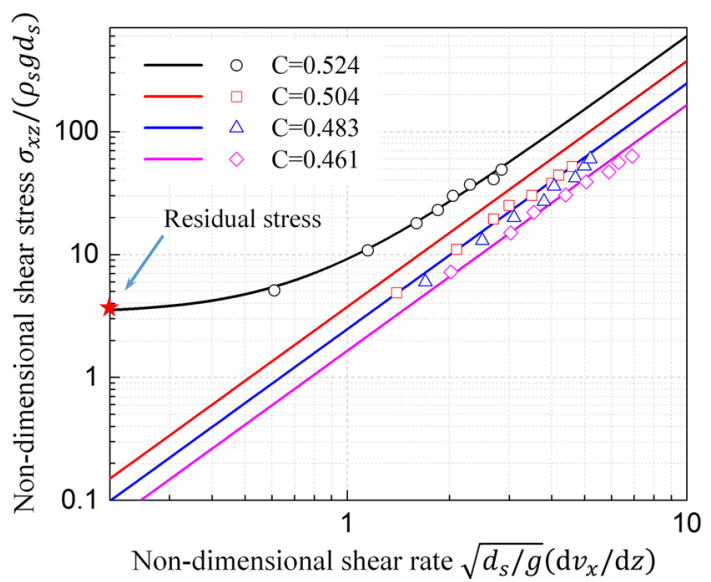

Fig. 4 Dry granular flow with different solid volume fraction: shear rate versus shear stress. The markers denote experimental data from [44]

from particle density $\rho_{s}$, particle diameter $d_{s}$ and volume fraction $C$. The obtained viscous coefficient $k_{\mathrm{v}}$ for the four simulations is also listed in Table 3. Since $\rho_{s}$ and $d_{s}$ are constants for a granular material, the solid volume fraction (void ratio) is significant to the granular flowing property as it has great influence on $k_{\mathrm{v}}$.

The shear and normal stresses developed in rapid shearing are shown in Figs. 4 and 5. Following Savage and Sayed [44], the non-dimensional shear rate and stress are employed. The experimental results are well reproduced by the numerical simulations in general. The agreement for the normal stress is slightly better than that for the shear stress. From Table 3, we know that frictional contacts vanish in the three simulations with loose specimens provided the shear strain be large. As a consequence, only particle collision contributes to the stress development. This can also be observed from Figs. 4 and 5. The shear rate-stress relationship is linear in the logarithm

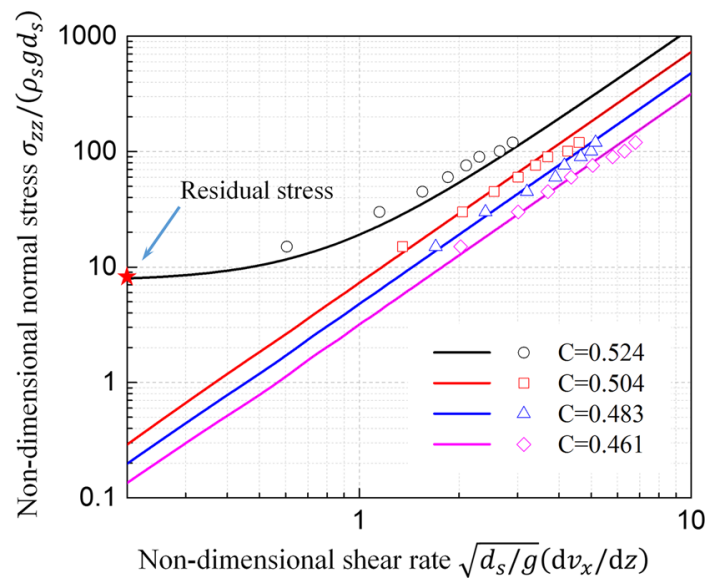

Fig. 5 Dry granular flow with different solid volume fraction: shear rate versus normal stress. The markers denote experimental data from [44] coordinates for the three loose specimens, consistent with the quadratic relationship used to calculate the dynamic stress part. On the other hand, particular attention should be paid to the case $C=0.524$ with dense specimen. In this case, the frictional contacts and collisions contribute to the stress development simultaneously. At low shear rate, frictional contact is significant, resulting in nonzero residual stress. As the shear rate increases, the stress contributed from collision becomes bigger and bigger. At high shear rate, although the magnitude of the frictional stress is unchanged, its proportion in the total stress declines. From this element test, it can be seen that the evolution of stress from the quasi-static state to rapid shear flow is correctly modelled.

\section{Application to boundary value problems}

Two element tests are performed in Sect. 4, where the velocity fields are prescribed to reproduce uniform deformation conditions. The tests give preliminary validation of our unified approach. In this section, the proposed unified modelling method is applied to real granular flow problems.

\subsection{Granular flow on an incline}

The problem of a granular mass on an incline is considered, as shown in Fig. 6. The inclination is $\theta$, the granular mass has a free surface and is subjected to gravity. The total height of the granular mass is $H$. In this section, we study characteristics of the granular flow on different inclinations.

\subsubsection{Analysis based on the unified model}

For a static granular mass or a steady flow, the stress components at arbitrary height $z$ should be hydrostatic

$\sigma_{z z}=\rho g(H-z) \cos \theta, \quad \sigma_{x z}=\rho g(H-z) \sin \theta$

where $\rho$ is the bulk density. Now we proceed to calculate the actual shear stress provided by the frictional contact in

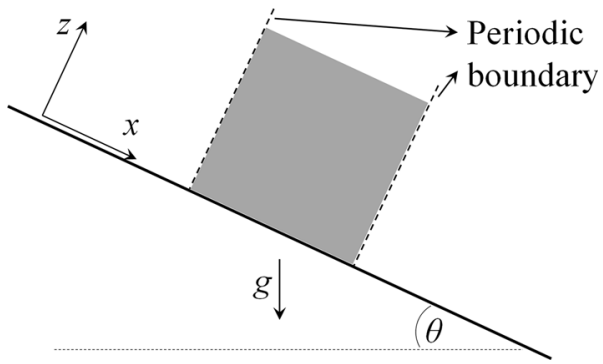

Fig. 6 Sketch-up of a granular flow on an inclined plate 
the plane parallel to the incline. The failure criterion in the hypoplastic model Eq. (3) is of the rounded Mohr-Coulomb type. Therefore, the failure of the material approximately follows the Mohr-Coulomb friction law. For an infinite steady granular flow on the incline, we assume that the failure slip lines are parallel to the plane. Therefore, the maximum shear stress provided by the hypoplastic model can be estimated as $\sigma_{x z}^{h}=\sigma_{z z}^{h} \tan \phi$, where $\phi$ is the granular internal friction angle. For the static state, the dynamic stress part can be neglected. Therefore, the balance in the $z$ direction requires $\sigma_{z z}=\sigma_{z z}^{h}=\rho g(H-z) \cos \theta$. As a result, the condition for static state is $\sigma_{x z}=\sigma_{x z}^{h} \geq \rho g(H-z) \sin \theta$, which means $\theta \leq \phi$.

When $\theta>\phi$, the granular mass starts to flow, generating a dynamic stress part. Under steady flow condition, by examining the force balance we have

$$
\begin{aligned}
& \sigma_{z z}=\rho g(H-z) \cos \theta=\sigma_{z z}^{h}+\sigma_{z z}^{v}, \quad \sigma_{z z}^{v}=4 k_{\mathrm{v}} \dot{\varepsilon}_{x z}^{2} / \tan \alpha_{i}, \\
& \sigma_{z z}^{h}=\sigma_{z z}-\sigma_{z z}^{v}
\end{aligned}
$$$$
\sigma_{x z}=\rho g(H-z) \sin \theta=\sigma_{x z}^{h}+\sigma_{x z}^{v}=\sigma_{z z}^{h} \tan \phi+4 k_{\mathrm{v}} \dot{\varepsilon}_{x z}^{2}
$$

In the steady granular flow, the stress consists of the contributions from a frictional contact part and a collision part, described by the hypoplastic model and the Bagnold-type rheology, respectively. Owing to the steady flow assumption, the strain rate tensor has only one nonzero component $\dot{\varepsilon}_{x z}=0.5 \partial v_{x} / \partial z$.

Based on Eqs. (32) and (33), we obtain the velocity profile by solving a differential equation

$$
\begin{gathered}
A\left(\frac{\partial v_{x}}{\partial z}\right)^{2}-B(H-z)=0, \quad A=\left(1-\tan \phi / \tan \alpha_{i}\right) k_{\mathrm{v}}, \\
B=\rho g(\sin \theta-\cos \theta \tan \phi)
\end{gathered}
$$

Solving the above equation, we have the velocity profile

$$
v_{x}(z)=\frac{2}{3} \sqrt{B / A}\left(H^{3 / 2}-(H-z)^{3 / 2}\right)
$$

and its derivative

$$
\frac{\partial v_{x}}{\partial z}=\sqrt{B / A}(H-z)^{1 / 2}
$$

The velocity profile, evolving as $(H-z)^{3 / 2}$, corresponds to a Bagnold profile [11]. Once the velocity profile is obtained, the hypoplastic and dynamic stress parts can be calculated from Eqs. (32) and (33). Eqs. (35) and (36) show that the velocity and shear rate increase with the inclination $\theta$. Therefore, with increasing $\theta$, the normal and shear stresses caused by collision become more significant. The increase in normal stress gives rise to a trend of dilation, tending to reduce the possibility of frictional contact thus resulting in a decrease in the frictional (hypoplastic) stress part.

If we define a phenomenological frictional coefficient $\mu=\sigma_{x z} / \sigma_{z z}$, in steady shearing flow we can observe that the coefficient $\mu$ increases with $\theta$ because the force balance requires that $\mu=\tan \theta$. This increase in the phenomenological frictional coefficient is found in many tests, as well as described by $\mu(I)$ model by Jop et al. [24]. According to classical explanations, this increase is the result of granular dilation [1]. In the present work, this dilation can be properly described by the combination of the hypoplastic model and Bagnold-type rheology. The maximum inclination $\theta^{*}$, beyond which a steady shearing flow is impossible, corresponds to a state of gasification where frictional (hypoplastic) stresses disappear. To find this critical angle, we have the following analysis. The force balance requires that $\sigma_{x z} / \sigma_{z z}=\tan \theta$; the frictional behaviour described by hypoplastic model is $\sigma_{x z}^{h} / \sigma_{z z}^{h}=\tan \phi$; in high speed shear, the pure collision of granular material results to the relation $\sigma_{x z}^{v} / \sigma_{z z}^{v}=\tan \alpha_{i}$. Therefore we have

$$
\begin{aligned}
\tan \theta & =\frac{\sigma_{x z}}{\sigma_{z z}}=\frac{\sigma_{x z}^{h}+\sigma_{x z}^{v}}{\sigma_{z z}}=\frac{\left(\sigma_{z z}-\sigma_{z z}^{v}\right) \tan \phi+\sigma_{z z}^{v} \tan \alpha_{i}}{\sigma_{z z}} \\
& =\tan \phi+\frac{\sigma_{z z}^{v}}{\sigma_{z z}}\left(\tan \alpha_{i}-\tan \phi\right)
\end{aligned}
$$

In steady granular flows, the range of $\sigma_{z z}^{v}$ is $0 \leq \sigma_{z z}^{v} \leq \sigma_{z z}$, we can therefore obtain the range of $\theta$

$\phi \leq \theta \leq \alpha_{i}$

Only in this range steady dense granular flows can be observed. Therefore, we have the critical inclination $\theta^{*}=\alpha_{i}$. When $\theta>\alpha_{i}$, frictional (hypoplastic) stress part disappears. The shear stress provided by granular collisions is insufficient to balance the driving force, so the granular mass accelerates continuously and reaches a gasified state. It is noteworthy that the obtained results are quite similar to that from $\mu(I)$ model [11], where the coefficients $\mu_{s}$ and $\mu_{l}$ correspond to $\tan \phi$ and $\tan \alpha_{i}$, respectively.

One important assumption in the above analysis is $\sigma_{x z}^{h} / \sigma_{z z}^{h}=\tan \phi$ at failure and subsequent flow state. When describing granular flows in the framework of depth-averaged equations, Savage and Hutter employ the same assumption [42]. However, some discrete simulations suggest that $\sigma_{x z}^{h} / \sigma_{z z}^{h}=\sin \phi[2,56]$, indicating a different failure slip line. Besides, the actual failure surface of the hypoplastic model is slightly different from that of MohrCoulomb model, which may result to minor violation of the frictional law. Nevertheless, the above analysis process is valid in principal, though the exact ratio between $\sigma_{x z}^{h}$ and $\sigma_{z z}^{h}$ may be put to further discussion. Considering 
separately the friction and collision in granular flow gives rise to interesting findings.

\subsubsection{Numerical modelling}

The numerical setups for the simulation of the steady granular flow are shown in Fig. 7. The height of the granular body is $H=0.03 \mathrm{~m}$. A square granular body is modelled with periodic boundary condition. The material constitutive parameters are given in Table 4. The hypoplastic parameters $c_{1} \sim c_{4}$ correspond to an internal friction angle $\phi=25^{\circ}$. The critical state parameters and initial void ratio are chosen such that the residual friction angle $\phi_{r}=\phi$, i.e. no softening or hardening occurs. Other physical constants are granular diameter $d_{s}=1.1 \mathrm{~mm}$, particle density $\rho_{s}=1095 \mathrm{~kg} / \mathrm{m}^{3}$. Three simulations with varied slope angle $\theta=26.0^{\circ}, 27.5^{\circ}$ and $30.0^{\circ}$, are performed. These angles lie in the range of $\phi<\theta<\alpha_{i}$, steady granular flows are therefore expected (see Sect. 5.1.1). All particles are at rest at the beginning of simulation. As $\theta>\phi$, the shear force provided by particle friction cannot balance the driving force. The granular mass starts to flow, the dynamic stress part begins to develop. The two stress parts evolve simultaneously, adjust continuously according to the flowing state, loading conditions and boundary conditions. Eventually, the flow reaches a steady shearing state.

To compare flows with different inclinations, the following dimensionless variables are used in the analysis: $z^{*}=z / H ; v_{x}^{*}=\left(v_{x} / \sqrt{g H}\right)\left(d_{s} / H\right) ; \sigma^{*}=\sigma /(\rho g H)$. Steady granular flows are observed in all the tested inclinations in numerical simulation. The velocity profiles are given in

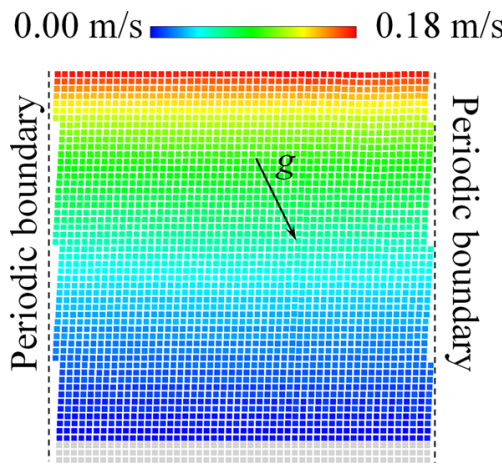

Fig. 7 Numerical setups for the simulation of steady granular flow. The colour scale represents the velocity field in $\theta=26^{\circ}$
Fig. 8. The analytical solutions in Sect. 5.1.1 are used as the reference. The velocity changes in height show clearly Bagnold profile. For all three inclinations, the numerical results are in good agreement with the analytical solutions. Figures 9 and 10 give the frictional contact (hypoplastic) and collisional stresses, respectively. A systematic agreement between the analytical and numerical results is observed. The discrepancy is pronounced close to the bottom, particularly on the profiles of frictional contact stress. It can be found that as the inclination increases, the frictional contact becomes less significant, and collisional stress turns into the dominant one. The two stress parts evolve simultaneously, automatically balancing the external loading. The presented results can be regarded as a validation of the numerical implementation of the unified model within the SPH method.

\subsection{Collapse of a granular pile}

The collapse of a granular pile on a flat surface is studied in this section. This problem has well-defined initial and boundary conditions and has been studied experimentally and numerically [7, 36, 29]. Previously numerical simulations often apply rate-independent constitutive models, thus do not consider the dynamic effect in granular flows.

The simulation is performed under plain strain condition. The initial geometry and boundary conditions are shown in Fig. 11. A pile of granular material with $10 \mathrm{~cm}$ height and $20 \mathrm{~cm}$ width is initially under static state. Similar to a dam-break problem in fluid dynamics, after suddenly releasing the confining gate, the granular pile starts to collapse. The material properties are the same as those used in Sect. 5.1 except $c_{1} \sim c_{4}$. The parameters $c_{1} \sim c_{4}$ are taken as $c_{1}=-50, c_{2}=-832.1, c_{3}=-832.1$ and $c_{4}=2369.9$, which correspond to a friction angle of $22^{\circ}$. The initial particle spacing is taken as $0.002 \mathrm{~m}$, giving rise to approximately 5000 particles.

From Eq. (12), we find that an equivalent viscosity can be defined as $\eta=2 k_{\mathrm{v}} \sqrt{\left\|\dot{\boldsymbol{\varepsilon}}^{*}\right\|}$. The equivalent viscosity is not only dependent on material properties, but also on the granular kinematics. It is related to both viscous shear stress and dispersive pressure. In quasi-static region, the equivalent viscosity is negligibly small, thus it can be used to distinguish the solid-like and fluid-like regions. Figure 12 shows the process of the collapse together with the evolution of the equivalent viscosity $\eta$. The flowing area

Table 4 Material parameters for the granular material on a inclined plate

\begin{tabular}{llllllllllll}
\hline $\begin{array}{l}c_{1} \\
(-)\end{array}$ & $\begin{array}{l}c_{2} \\
(-)\end{array}$ & $\begin{array}{l}c_{3} \\
(-)\end{array}$ & $\begin{array}{l}c_{4} \\
(-)\end{array}$ & $\begin{array}{l}e_{\min } \\
(-)\end{array}$ & $\begin{array}{l}p_{1} \\
(-)\end{array}$ & $\begin{array}{l}p_{2} \\
(-)\end{array}$ & $\begin{array}{l}p_{3} \\
\left(\mathrm{kPa}^{-1}\right)\end{array}$ & $\begin{array}{l}q_{1} \\
(-)\end{array}$ & $\begin{array}{l}q_{2} \\
(-)\end{array}$ & $\begin{array}{l}q_{3} \\
\left(\mathrm{kPa}^{-1}\right)\end{array}$ & $\begin{array}{l}k_{\mathrm{v}} \\
(-)\end{array}$ \\
\hline-66.7 & -832.9 & -832.9 & 1594.5 & 0.597 & 0.53 & 0.45 & -0.0011 & 1.0 & -0.4 & -0.0001 & 0.1 \\
\hline
\end{tabular}




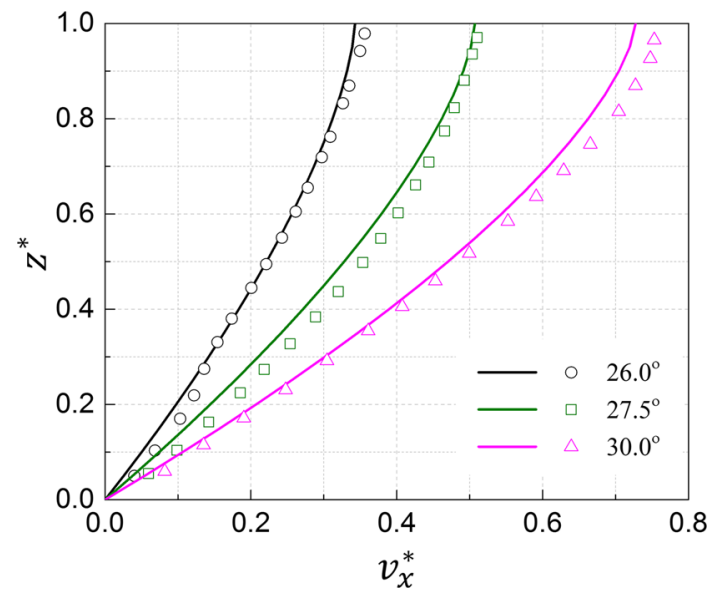

Fig. 8 Velocity profiles: lines indicate reference solutions calculated from Eq. (35) and markers are numerical results

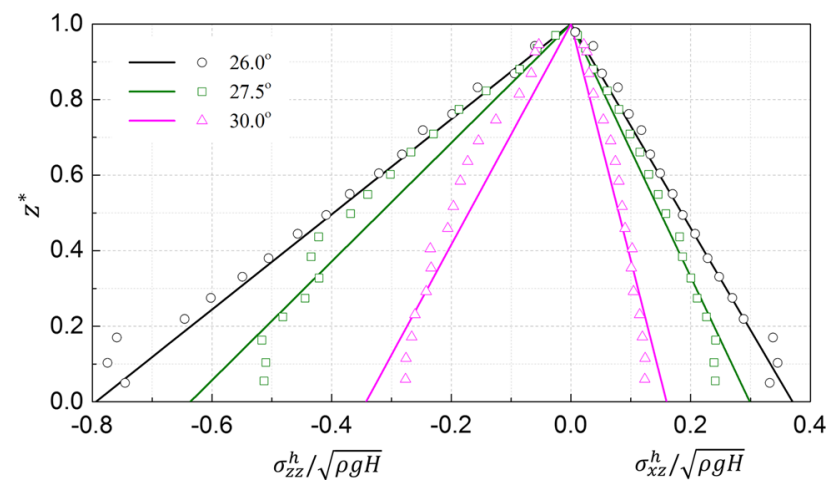

Fig. 9 Normalized analytical and numerical frictional contact (hypoplastic) stress. Normal stress is in the left (negative part) and shear stress in the right (positive part)

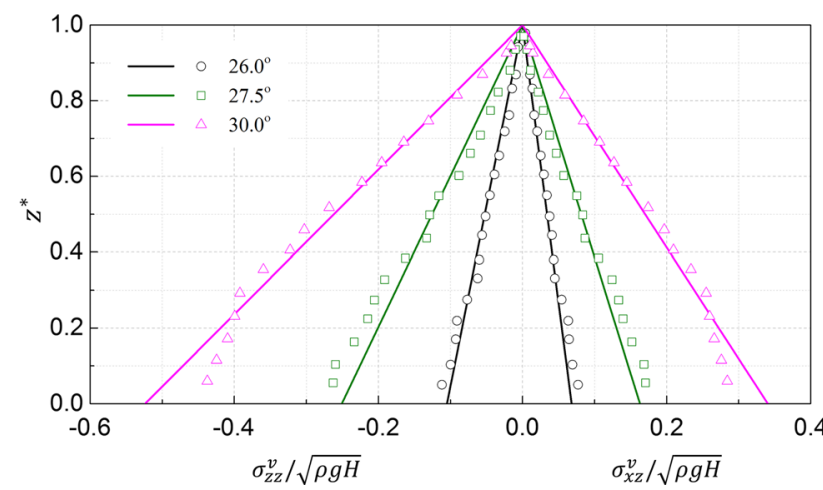

Fig. 10 Normalized analytical and numerical collisional stress. Normal stress is in the left (negative part) and shear stress in the right (positive part)

propagates from right to left. There exists a slip plane above which the particle moves and below which particles are generally at rest. The equivalent viscosity helps us easily identify the flowing state. In the flow regions, significant parts of stress components are developed by

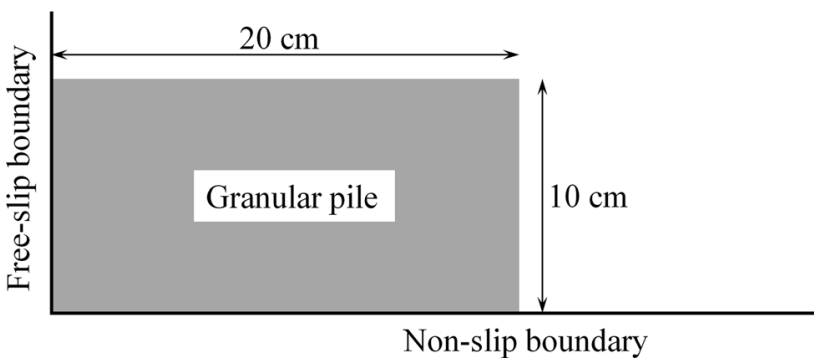

Fig. 11 Initial geometry and boundary conditions

particle collisions. To demonstrate the evolution of dynamic stress in the collapse, in Fig. 13, we show the percentage of the dynamic part of stress in the total vertical stress component $\left(\sigma_{z z}^{v} /\left(\sigma_{z z}^{v}+\sigma_{z z}^{h}\right) \times 100 \%\right)$ at three different particles throughout the simulation. From the initial locations of the three particles, we can see particle A and B are in the potential flow area with A closer to the surface, particle $\mathrm{C}$ is deep inside the granular body, subjected to less deformation in the collapse. In the collapse, dynamic stresses at particle A and B are significant, indicating partial fluid-like behaviours. For a short period, the dynamic stress accounts for the whole stress (percentage reaches $100 \%$ ), which means the granular material behaves like a pure Bagnold-type fluid. With collapse coming to its end, the significance of dynamic part reduces, the granular material behaves more and more like solid. Once granular mass finally reaches static packing, dynamic stress part disappears and stress components are all developed by frictional contacts. Comparison between particles A and B shows that dynamic stress is usually larger at particle $\mathrm{A}$, indicating particles near the right surface are more fluid-like in the collapse. At particle C deep in the granular body, the dynamic stress components are very close to zero. The material is solid-like and does not undergo fluid-like flow.

The proposed unified model bears essential difference with Bingham model or pure Bagnold-type model. It can account for the rate-independent solid state accurately with its hypoplastic component. Material behaviours inside yield surface are well-defined. In simulations with the Bingham model or pure Bagnold-type models, materials will never reach the final static state, creeping is usually observed. However, in simulations with the unified model, material can reach the final static state because the final solid-state is considered with the hypoplastic model. Figure 14 gives the evolution of runout distance in time. It is observed that the granular pile reaches final state in about $0.5 \mathrm{~s}$. From then on the granular media is at rest, no creep is observed.

It is well known that SPH suffers the so-called shortlength-scale-noise, which leads to stress fluctuations in areas with large deformation [33]. Several methods are 


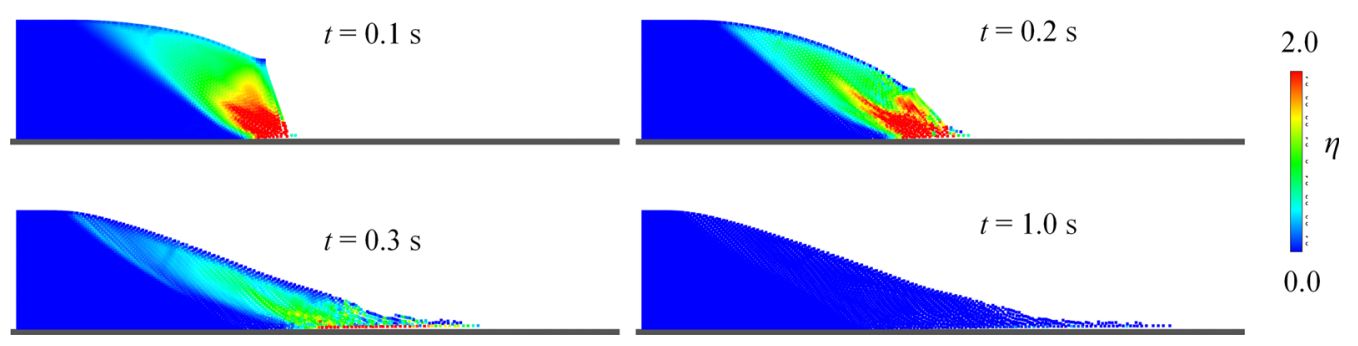

Fig. 12 The collapse process of the granular pile. The figures are coloured by equivalent viscosity $\eta$

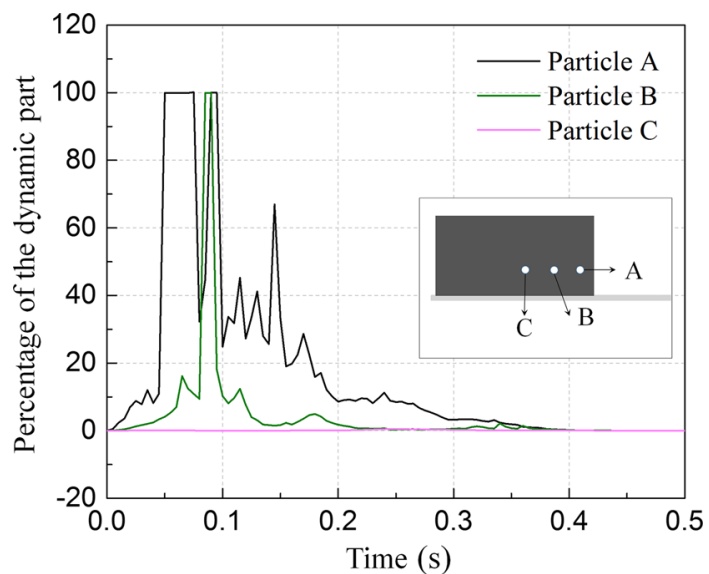

Fig. 13 Change in percentage of dynamic stress part in the total stress at three particles

proposed to mitigate this deficiency, e.g. artificial viscosity and stress regularization. In this paper, only artificial viscosity is used. The stress results at the final state are shown in Fig. 15. In areas without particle rearrangement, very smooth stress distributions are obtained. However, in regions with large deformation, stress oscillations are observed. Fortunately, it is reported that the kinematics obtained through SPH are generally satisfactory even with stress oscillation [33]. This finding is also confirmed by our simulations. Nevertheless, the stress accuracy is a challenge for SPH and should be put to further study.

\subsection{Granular flow in a rotating drum}

A granular mass rotating in a drum is modelled in this section. The material properties are the same as those used in Sect. 5.1. The numerical setup is shown in Fig. 16. The granular flows in the rotating drums are surface flows over the static grains. The granular quasi-static and dynamic flowing states coexist in this configuration. The flows in rotating drums are inhomogeneous in flow depth and inclination. Therefore, analytical solutions are usually unavailable.

Three simulations with different angular velocities $\omega=0.5,1.0$ and $2.0 \mathrm{~s}^{-1}$ are performed. The rotating process of $\omega=1.0 \mathrm{~s}^{-1}$ is shown in Fig. 17. At first the

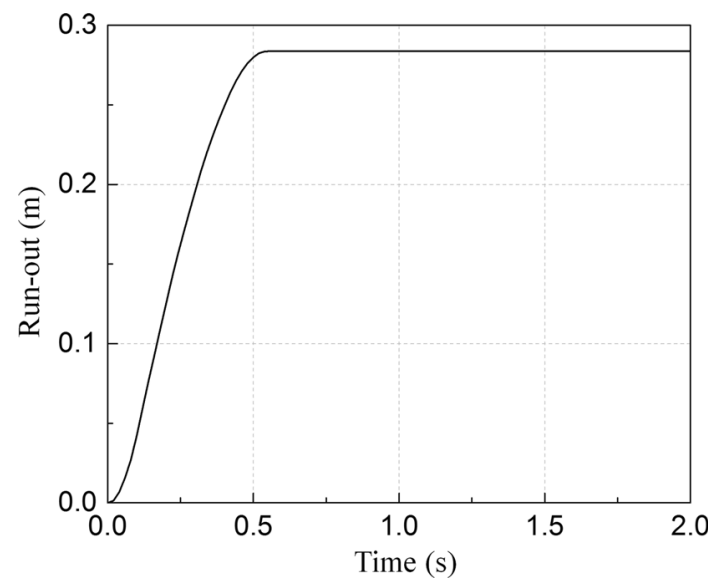

Fig. 14 Evolution of runout distance

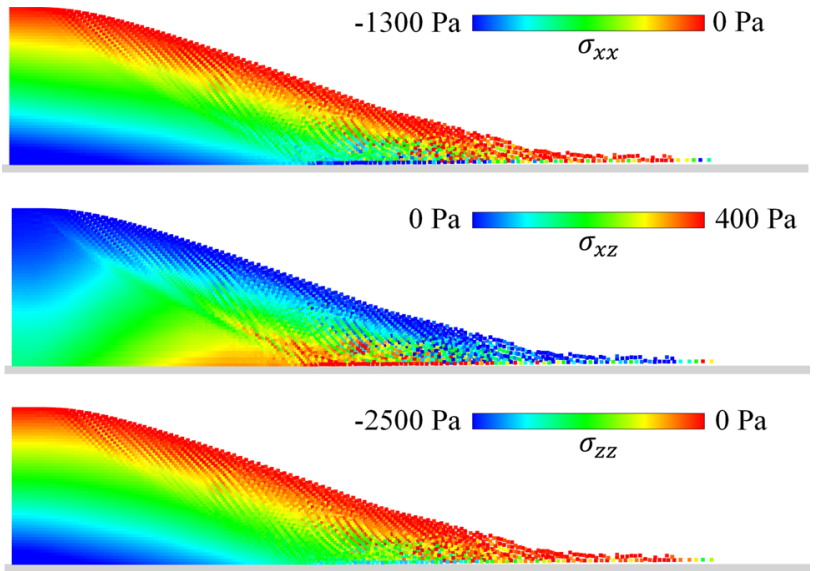

Fig. 15 Stress distributions in the final state

granular mass moves with the drum like a solid block without any deformation. As the surface slope angle grows, failure occurs in the granular body. As a consequence, shear band develops, and the first avalanche takes place. This first avalanche is followed by several small avalanches, and eventually a steady surface granular flow is observed. Below the surface flow, the granular remains quasi-static and moves with the drum as a solid block. This process is further confirmed by the evolution of the maximum surface speed, as shown in Fig. 18. The velocity evolutions under different rotating angular speeds are 
similar. The peaks in Fig. 18 mark the first avalanche. As expected, the appearance of this critical event is delayed with the decrease of the rotating velocity of the drum. In each simulation, a steady flow state is achieved after several seconds of rotating.

A local coordinate as shown in Fig. 16b is used, and the flow characteristics along direction $z$ are studied. $z_{y}$ is the height where flow state changes and $z_{s}$ is the height of the flow surface; $h=z_{s}-z_{y}$ is the flow depth. In the range of $z<z_{y}$ the granular mass behaves solid-like, so the velocity profile is linear. The steady velocity profiles in the dynamic flowing layer are given in Fig. 19. On a middle cross section, the velocity direction pointing down the slope is defined as positive. The velocity profiles for different rotating speeds are similar in shape but with different magnitudes. In experiments [31], similar velocity profiles are observed. The simulated flow rates, flow depths and average inclinations are summarized in Table 5. In our flow configuration, the flow rate is calculated by $Q=0.5 \omega\left[R^{2}-\left(R-H_{0}\right)^{2}\right]$, where $R=D / 2$ is the drum radius. It is found that the flow depth $h$ and average inclination $\theta$ changes almost linearly with respect to the flow rate $Q$. This observation is also well collaborated with the experimental results [31].

In the drum the surface flow moves over the solid-like granular body, so both static and flow states coexist in the computational domain. In the simulations, the dynamic part is always taken into account; however, in the quasi-static zone the calculated collisional stress is negligible that the dynamic part has no realistic effect on the mechanical response of the material. In the beginning, there is no flow and the granular behaves like solid. Phenomena observed in solids, such as non-isotropic stress tensor and shear band, are reproduced in the simulations. As shown in Fig. 20, in the steady flow state the flowing zone, where
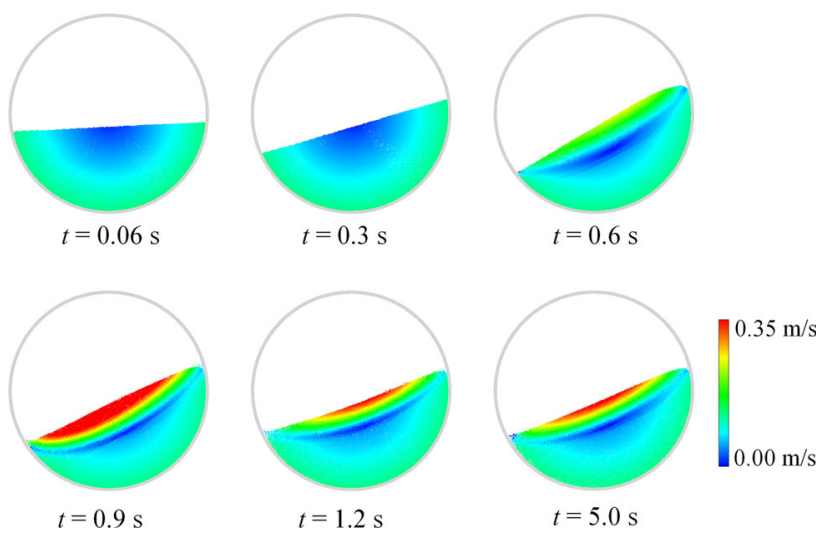

Fig. 17 The developing process of steady surface flow in the rotating drum, $\omega=1.0 \mathrm{~s}^{-1}$

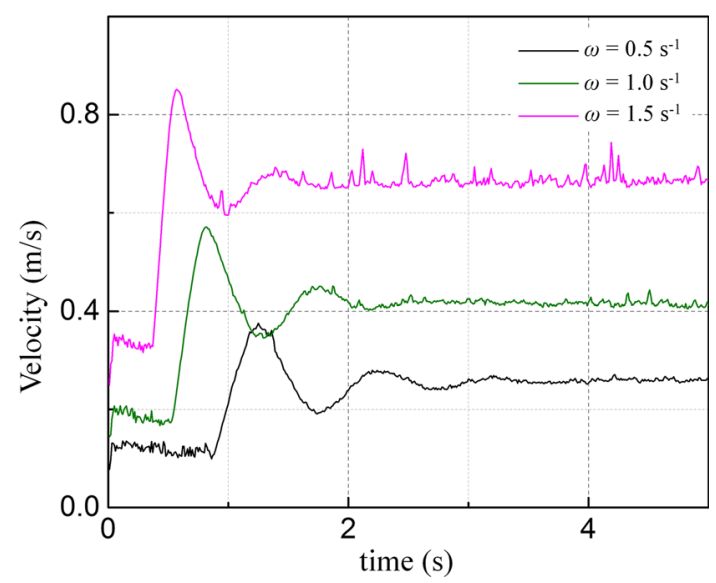

Fig. 18 Evolution of maximum surface velocity

dynamic stress part takes effect, can be marked by the equivalent viscosity defined in Sect. 5.2. In the zones marked blue, the equivalent viscosity is almost zero. Therefore, the dynamic part of the constitutive model has

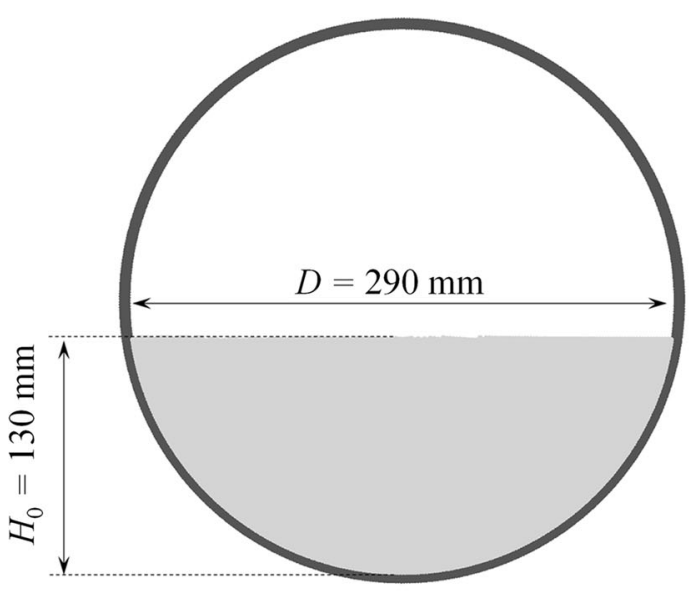

(a)

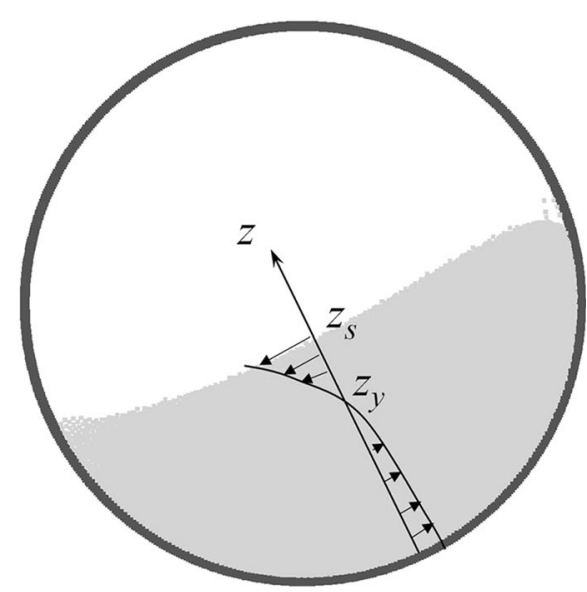

(b)

Fig. 16 Numerical set-ups for the simulation of granular in a rotating drum: a initial state; b steady flow state 


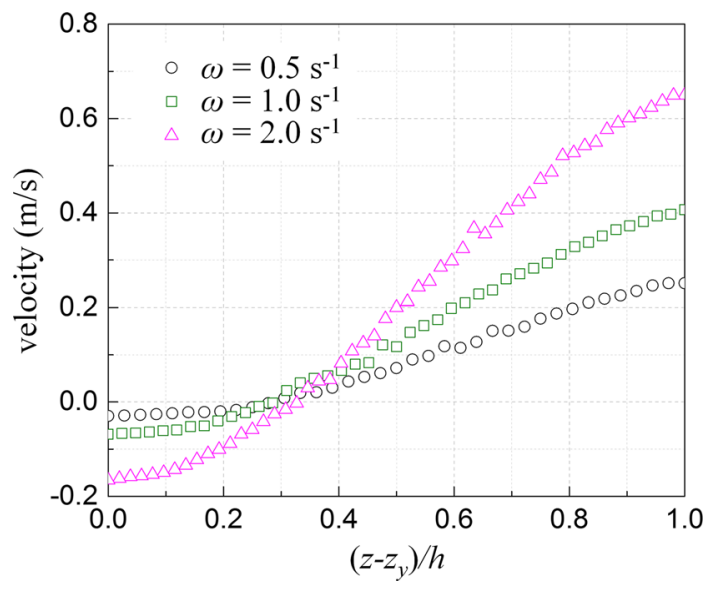

Fig. 19 Velocity profiles in the flowing layer

Table 5 Summary of the numerical modelling of rotating drum

\begin{tabular}{llll}
\hline Rotating speed $\omega\left(\mathrm{s}^{-1}\right)$ & 0.5 & 1.0 & 2.0 \\
Flow rate $Q\left(\mathrm{~m}^{2} / \mathrm{s}\right)$ & 0.0052 & 0.0104 & 0.0208 \\
Flowing depth $h(\mathrm{~m})$ & 0.046 & 0.054 & 0.065 \\
Average inclination $\theta\left(^{\circ}\right)$ & 21.48 & 22.43 & 24.81 \\
\hline
\end{tabular}

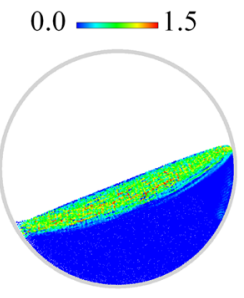

(a)

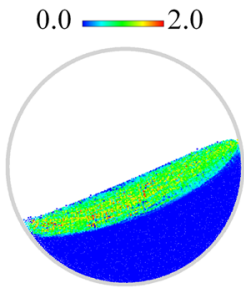

(b)

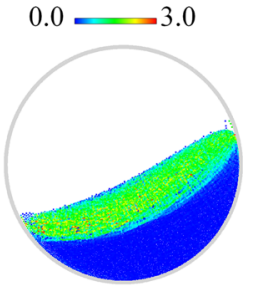

(c)
Fig. 20 Quasi-static and flowing regions in the drum: a $\omega=0.5 \mathrm{~s}^{-1}$; b $\omega=1.0 \mathrm{~s}^{-1} ; \mathbf{c} \omega=2.0 \mathrm{~s}^{-1}$. The figures are coloured by the equivalent viscosity $\eta$

no realistic effect. As a result, only the solid-like behaviours are described by the hypoplastic model. From the simulations, we can see that the solid-like and flow behaviours of granular material are successfully modelled with the unified approach. The explicit determination of the solid/flow states is unnecessary. The solid/fluid transition is achieved naturally in the computation. We do not make use of concepts such as yield stress or flow initiation criterion. Therefore, the numerical implementation is greatly simplified.

\section{Conclusions}

This paper presents a unified framework for modelling granular media. The hypoplastic model and Bagnold-type relation, respectively, employed to consider the granular frictional contact and collision, are combined to obtain a complete constitutive model for the entire deformation and flow regime of granular media. The unified model makes no use of explicit determination of solid/flow state or flow initiation criterion. It covers the whole spectrum of granular state from quasi-static motion to rapid granular flow. This conversion of motion state is achieved by the coupled evolution of the frictional contact and collision stress parts. The solid-like and fluid-like behaviours of granular materials, as well as the transition between the solid-like and fluid-like states, are well described by the unified constitutive model.

The SPH is proved ideal for modelling both solid-like and fluid-like behaviours within a consistent numerical scheme. The numerical simulation of two element tests shows that the unified approach captures the salient feature of the quasistatic and flowing states of granular materials. We proceed to study three boundary value problems: two granular flows, one on an incline the other in a rotating drum, and a granular pile collapse problem. The following observations are made: (1) In the case of granular flow down an inclined plane, we have obtained a range of inclinations during which a steady dense granular flow is observed. The numerical simulation agrees well with the analytical solution derived from the unified model. Moreover, the solutions to this problem in the present model are well collaborated with those from $\mu(I)$ model $[24,11]$. (2) For the granular pile collapse and the granular flow in rotating drum, the numerical results show wealth of various behaviours, i.e. quasi-static motion, shear band, flow initiation, fully developed granular flow and granular deposition.

The numerical results in this paper are promising to handle the complex behaviour of granular flow in a consistent numerical model. We will apply the unified approach to bulk solids handling in industry and debris flow in nature. However, some aspects, such as particle segregation and hydro-mechanical coupling, still need further investigations to be considered in the unified framework.

Acknowledgments Open access funding provided by University of Natural Resources and Life Sciences Vienna (BOKU). The authors acknowledge the funding from the European Commission under its People Programme (Marie Curie Actions) of the Seventh Framework Programme FP7/2007-2013/ under Research Executive Agency Grant agreement No. 289911 under title: Multiscale Modelling of Landslides and Debris Flows. The first author wishes to acknowledge the financial support from the Otto Pregl Foundation for Fundamental Geotechnical Research in Vienna.

Open Access This article is distributed under the terms of the Creative Commons Attribution 4.0 International License (http:// creativecommons.org/licenses/by/4.0/), which permits unrestricted use, distribution, and reproduction in any medium, provided you give appropriate credit to the original author(s) and the source, provide a 
link to the Creative Commons license, and indicate if changes were made.

\section{References}

1. Andrade JE, Chen QS, Le PH, Avila CF, Evans TM (2012) On the rheology of dilative granular media: bridging solid-and fluidlike behavior. J Mech Phys Solids 60(6):1122-1136

2. Andreotti B, Forterre Y (2013) Granular media: between fluid and solid. Cambridge University Press, Cambridge

3. Bagnold RA (1956) The flow of cohesionless grains in fluids. Philos Trans R Soc Lond Math Phys Eng Sci 249(964):235-297

4. Bagnold RA (1954) Experiments on a gravity-free dispersion of large solid spheres in a Newtonian fluid under shear. In: Proceedings of the Royal Society of London A: Mathematical, Physical and Engineering Sciences, vol 225. The Royal Society, pp 49-63

5. Barker T, Schaeffer DG, Bohorquez P, Gray JMNT (2015) Wellposed and ill-posed behaviour of $\mu$-(I) rheology for granular flow. J Fluid Mech 779:794-818

6. Bonet J, Lok T (1999) Variational and momentum preservation aspects of Smooth Particle Hydrodynamic formulations. Computer Methods Appl Mech Eng 180(1):97-115

7. Bui HH, Fukagawa R, Sako K, Ohno S (2008) Lagrangian meshfree particles method ( $\mathrm{SPH}$ ) for large deformation and failure flows of geomaterial using elastic-plastic soil constitutive model. Int J Numer Anal Methods Geomech 32(12):1537-1570

8. Campbell CS (1990) Rapid granular flows. Annu Rev Fluid Mech 22(1):57-90

9. Cante J, Davalos C, Hernandez JA, Oliver J, Jonsén P, Gustafsson G, Häggblad H (2014) PFEM-based modeling of industrial granular flows. Comput Part Mech 1(1):47-70

10. Castro G (1969) Liquefaction of sands. Harvard University, Cambridge

11. Chambon G, Bouvarel R, Laigle D, Naaim M (2011) Numerical simulations of granular free-surface flows using Smoothed Particle Hydrodynamics. J Non-Newton Fluid Mech 166(12):698-712

12. Chen W, Qiu T (2011) Numerical simulations for large deformation of granular materials using Smoothed Particle Hydrodynamics method. Int J Geomech 12(2):127-135

13. Chen JF, Rotter JM, Ooi JY, Zhong Z (2007) Correlation between the flow pattern and wall pressures in a full scale experimental silo. Eng Struct 29(9):2308-2320

14. Cleary PW, Sawley ML (2002) DEM modelling of industrial granular flows: 3D case studies and the effect of particle shape on hopper discharge. Appl Math Model 26(2):89-111

15. Coussot P, Raynaud JS, Bertrand F, Moucheront P, Guilbaud JP, Huynh HT, Jarny S, Lesueur D (2002) Coexistence of liquid and solid phases in flowing soft-glassy materials. Phys Rev Lett 88(21):218301

16. Darve F (1994) Stability and uniqueness in geomaterials constitutive modelling. In: Chambon et al (eds) Localization and bifurcation theory for soils and rocks. Balkema, Rotterdam, pp 73-88

17. Dávalos C, Cante J, Hernández JA, Oliver J (2015) On the numerical modeling of granular material flows via the Particle Finite Element Method (PFEM). Int J Solids Struct 71:99-125

18. De Alba PA, Chan CK, Seed HB (1976) Sand liquefaction in large-scale simple shear tests. J Geotech Eng Div 102(9):909-927

19. Dehnen W, Aly H (2012) Improving convergence in Smoothed Particle Hydrodynamics simulations without pairing instability. Mon Not R Astron Soc 425(2):1068-1082
20. Elaskar SA, Godoy LA (1998) Constitutive relations for compressible granular materials using non-Newtonian fluid mechanics. Int J Mech Sci 40(10):1001-1018

21. Gomez-Gesteira M, Rogers BD, Crespo AJC, Dalrymple RA, Narayanaswamy M, Dominguez JM (2012) SPHysics-development of a free-surface fluid solver-part 1: theory and formulations. Comput Geosci 48:289-299

22. Jaeger HM, Nagel SR, Behringer RP (1996) Granular solids, liquids, and gases. Rev Mod Phys 68(4):1259

23. Jenkins J, Savage SB (1983) A theory for the rapid flow of identical, smooth, nearly elastic, spherical particles. J Fluid Mech 130:187-202

24. Jop P, Forterre Y, Pouliquen O (2006) A constitutive law for dense granular flows. Nature 441(7094):727-730

25. Laouafa F, Darve F (2002) Modelling of slope failure by a material instability mechanism. Comput Geotech 29(4):301-325

26. Larese A, Rossi R, Onate E, Idelsohn SR (2012) A coupled PFEM-Eulerian approach for the solution of porous FSI problems. Comput Mech 50(6):805-819

27. Li Z, Kotronis P, Escoffier S, Tamagnini C (2015) A hypoplastic macroelement for single vertical piles in sand subject to threedimensional loading conditions. Acta Geotechnica 11:1-18

28. Liu MB, Liu GR (2010) Smoothed Particle Hydrodynamics (SPH): an overview and recent developments. Arch Comput Methods Eng 17(1):25-76

29. Lube G, Huppert HE, Sparks RSJ, Freundt A (2005) Collapses of two-dimensional granular columns. Phys Rev E 72(4):041301

30. Mast CM, Arduino P, Mackenzie-Helnwein P, Miller GR (2014) Simulating granular column collapse using the material point method. Acta Geotech 10:1-16

31. MiDi GDR (2004) On dense granular flows. Eur Phys J E 14(4):341-365

32. Nedderman RM, Tüzün U, Savage SB, Houlsby GT (1982) The flow of granular materials-I: discharge rates from hoppers. Chem Eng Sci 37(11):1597-1609

33. Nguyen CT, Nguyen CT, Bui HH, Nguyen GD, Fukagawa R (2016) A new sph-based approach to simulation of granular flows using viscous damping and stress regularization. Landslides 1-13. doi:10.1007/s10346-016-0681-y

34. Oger G, Doring M, Alessandrini B, Ferrant P (2007) An improved SPH method: Towards higher order convergence. J Comput Phys 225(2):1472-1492

35. Peng $\mathrm{C}, \mathrm{Wu} \mathrm{W}, \mathrm{Yu} \mathrm{HS}$, Wang $\mathrm{C}$ An improved wall boundary treatment for Smoothed Particle Hydrodynamics with applications in geomechanics. J Comput Phys (under review)

36. Peng C, Wu W, Yu HS, Wang C (2015) A SPH approach for large deformation analysis with hypoplastic constitutive model. Acta Geotech 10(6):703-717

37. Peng C, Wu W, Yu HS (2015) Hypoplastic constitutive model in sph. In: Oka F, Murakami A, Uzuoka R, Kimoto S (eds) Computer methods and recent advances in geomechanics - Proceedings of the 14th International Conference of International Association for Computer Methods and Recent Advances in Geomechanics, IACMAG 2014. CRC Press, pp 1863-1868

38. Prime N, Dufour F, Darve F (2013) Unified model for geomaterial solid/fluid states and the transition in between. J Eng Mech 140(6):04014031

39. Prime N, Dufour F, Darve F (2014) Solid-fluid transition modelling in geomaterials and application to a mudflow interacting with an obstacle. Int $\mathrm{J}$ Numer Anal Methods Geomech 38(13):1341-1361

40. Pudasaini SP (2012) A general two-phase debris flow model. J Geophys Res 117:F03010. doi:10.1029/2011JF002186

41. Savage SB (1979) Gravity flow of cohesionless granular materials in chutes and channels. J Fluid Mech 92(1):53-96 
42. Savage SB, Hutter K (1989) The motion of a finite mass of granular material down a rough incline. $J$ Fluid Mech 199:177-215

43. Savage SB, Nedderman RM, Tüzün U, Housby GT (1983) The flow of granular materials-III. Chem Eng Sci 38(2):189-195

44. Savage SB, Sayed M (1984) Stresses developed by dry cohesionless granular materials sheared in an annular shear cell. J Fluid Mech 142:391-430

45. Stutz H, Mašín D, Wuttke F (2016) Enhancement of a hypoplastic model for granular soil-structure interface behaviour. Acta Geotech 1-13. doi:10.1007/s11440-016-0440-1

46. Tankeo M, Richard P, Canot É (2013) Analytical solution of the $\mu$-(I) rheology for fully developed granular flows in simple configurations. Granul Matter 15(6):881-891

47. Teufelsbauer H, Wang Y, Chiou MC, Wu W (2009) Flow-obstacle interaction in rapid granular avalanches: DEM simulation and comparison with experiment. Granul Matter 11(4):209-220

48. Wang Y Hutter K (2001) Granular material theories revisited. In: Geomorphological fluid mechanics. Springer, pp 79-107

49. Wendland H (1995) Piecewise polynomial, positive definite and compactly supported radial functions of minimal degree. Adv Comput Math 4(1):389-396
50. Wu W, Bauer E (1994) A simple hypoplastic constitutive model for sand. Int J Numer Anal Methods Geomech 18(12):833-862

51. Wu W, Bauer E, Kolymbas D (1996) Hypoplastic constitutive model with critical state for granular materials. Mech Mater 23(1):45-69

52. Wu W, Niemunis A (1996) Failure criterion, flow rule and dissipation function derived from hypoplasticity. Mech Cohes Frict Mater 1(2):145-163

53. Wu W, Niemunis A (1997) Beyond failure in granular materials. Int J Numer Anal Methods Geomech 21(3):153-174

54. Wu W, Kolymbas D (2000) Hypoplasticity then and now. In: Constitutive modelling of granular materials. Springer, pp 57-105

55. Yoshimine M, Ishihara K, Wargas W (1998) Effects of principal stress direction and intermediate principal stress on undrained shear behaviour of sand. Soils Found 38(3):179-188

56. da Cruz F, Emam S, Prochnow M, Roux J, Chevoir F (2005) Rheophysics of dense granular materials: discrete simulation of plane shear flows. Phys Rev E 72(2):021309 